\title{
One-bit Decentralized Detection with a Rao Test for Multisensor Fusion
}

\author{
D. Ciuonzo, Student Member, IEEE, G. Papa, Student Member, IEEE, \\ G. Romano, Member, IEEE, P. Salvo Rossi, Senior Member, IEEE, and P. Willett, Fellow, IEEE
}

\begin{abstract}
In this letter we propose the Rao test as a simpler alternative to the generalized likelihood ratio test (GLRT) for multisensor fusion. We consider sensors observing an unknown deterministic parameter with symmetric and unimodal noise. A decision fusion center (DFC) receives quantized sensor observations through error-prone binary symmetric channels and makes a global decision. We analyze the optimal quantizer thresholds and we study the performance of the Rao test in comparison to the GLRT. Also, a theoretical comparison is made and asymptotic performance is derived in a scenario with homogeneous sensors. All the results are confirmed through simulations.
\end{abstract}

Index Terms-Decentralized detection, Rao test, threshold optimization, wireless sensor networks (WSNs).

\section{INTRODUCTION}

D ECENTRALIZED detection with wireless sensor networks (WSNs) has received close attention by the scientific community over the last decade. Each sensor, rather than sending its observed measurements, typically sends one bit of information about the estimated hypothesis to the decision fusion center (DFC), which makes a global decision. Such an approach is generally employed in order to satisfy stringent constraints on bandwidth and energy. In this context the optimal test (under Bayesian and Neyman-Pearson frameworks) at each sensor is well known to be a one-bit quantization of the local likelihood-ratio test (LRT). Unfortunately in most cases, due to a lack of signal knowledge, it is not possible to compute the local LRT at the generic sensor. Also, even when the sensors can compute their local LRT, the search for local quantization thresholds is well known to be exponentially complex [1], [2]. In such situations the raw measurement is directly quantized into a single bit of information; the DFC is then in charge of solving a composite hypothesis testing problem.

Some simple approaches have been based on the counting rule or channel-aware statistics, which neglect the dependence with respect to (w.r.t.) the unknown signal [3], [4], [5], [6]. On the other hand, in some particular scenarios the uniformly most powerful test is independent of the unknown parameters under the alternate hypothesis, which then do not need to be estimated [7]. Nonetheless, typically the fusion rule employed at the DFC is based on the generalized LRT (GLRT). GLRTbased fusion of quantized data was studied in [8], [9], for

D. Ciuonzo, G. Papa, G. Romano and P. Salvo Rossi are with the Department of Industrial and Information Engineering, Second University of Naples, Aversa (CE), Italy (e-mail: \{domenico.ciuonzo, giuseppe.papa, gianmarco.romano, pierluigi.salvorossi\}@unina2.it).

P. Willett is with the Department of Electrical and Computer Engineering, University of Connecticut, Storrs (CT), US (e-mail: willett@engr.uconn.edu). $\%$ This work was supported in part by the US Office of Naval Research under contract N00014-09-10613. detecting a source with unknown location and fusing conditionally dependent decisions, respectively. Recently in [10] the GLRT has been used to detect an unknown deterministic signal (in a decentralized fashion with quantized measurements and noisy communication channels of identical quality) and an asymptotically optimal threshold choice for the quantizer has been derived in the non-homogeneous sensor case (i.e. an additive Gaussian observation model with unequal variances).

The contributions of this letter are summarized hereinafter. We study the problem in [10] and we propose the Rao test as a computationally simpler alternative to the GLRT, since it does not require any estimation procedure; its closed form is obtained in the more general case of zero-mean noise with symmetric and unimodal pdf and non-identical bit-error probabilities (BEPs) on the communication channels. Also, we discuss the optimal choice of quantizer threshold for some pdfs of interest. Furthermore, the Rao test is compared to the GLRT through simulations showing that, in addition to sharing the same asymptotic distribution, it achieves practically the same performance for a finite number of sensors. This result becomes in fact theoretical coincidence in a scenario with homogeneous sensors; for the latter scenario a tighter asymptotic distribution of both tests is derived.

The letter is organized as follows: Sec. [II introduces the model; in Sec. [III we derive the Rao test and the corresponding optimal thresholds; in Sec. IV the GLR and Rao tests are compared analytically in a homogeneous scenario, while in Sec. D we confirm the results through simulations; in Sec. VI we draw some conclusions.

\section{Problem Statement}

The system model is described 1 as follows. We consider a binary hypothesis testing problem in which a collection of sensors $k \in \mathcal{K} \triangleq\{1, \ldots, K\}$ collaborate to detect the presence of an unknown deterministic parameter $\theta \in \mathbb{R}$. The problem can be summarized as follows:

$$
\begin{cases}\mathcal{H}_{0} \quad: \quad x_{k}=w_{k} \\ \mathcal{H}_{1}: & x_{k}=h_{k} \theta+w_{k}, \quad k \in \mathcal{K}\end{cases}
$$

\footnotetext{
${ }^{1}$ Notation - Lower-case bold letters denote vectors, with $a_{n}$ representing the $n$th element of $\boldsymbol{a}$; upper-case calligraphic letters, e.g. $\mathcal{A}$, denote finite sets; $\mathbb{E}\{\cdot\}, \operatorname{var}\{\cdot\}$ and $(\cdot)^{t}$ denote expectation, variance and transpose, respectively; $P(\cdot)$ and $p(\cdot)$ are used to denote probability mass functions (pmf) and probability density functions (pdf), respectively, while $P(\cdot \mid \cdot)$ and $p(\cdot \mid \cdot)$ their corresponding conditional counterparts; $\mathcal{N}\left(\mu, \sigma^{2}\right)$ denotes a normal distribution with mean $\mu$ and variance $\sigma^{2} ; \chi_{k}^{2}$ (resp. $\chi_{k}^{\prime 2}(\xi)$ ) denotes a chi-square (resp. a non-central chi-square) distribution with $k$ degrees of freedom (resp. and non-centrality parameter $\xi) ; \mathcal{U}(a, b)$ denotes a continuousvalued uniform distribution with support set $[a, b] ; \mathcal{L}(\mu, \beta)$ denotes a Laplace distribution with mean $\mu$ and scale parameter $\beta$; the symbols $\sim$ and $\stackrel{a}{\sim}$ mean "distributed as" and "asymptotically distributed as".
} 
where $x_{k} \in \mathbb{R}$ denotes the $k$ th sensor measurement, $h_{k} \in \mathbb{R}$ is a known observation coefficient and $w_{k} \in \mathbb{R}$ denotes the noise random variable (RV) with $\mathbb{E}\left\{w_{k}\right\}=0$ and unimodal symmetric $\operatorname{pd}$, denoted with $p_{w_{k}}(\cdot)$. Furthermore, the RVs $w_{k}$ are assumed mutually independent. It is worth noting that Eq. (11) refers to a two-sided test [11], where $\left\{\mathcal{H}_{0}, \mathcal{H}_{1}\right\}$ corresponds to $\left\{\theta=\theta_{0}, \theta \neq \theta_{0}\right\}$ (in our case $\theta_{0}=0$ ).

Also, to meet stringent bandwidth and power budgets in WSNs, the $k$ th sensor quantize $3 x_{k}$ into one bit of information, that is $b_{k} \triangleq u\left(x_{k}-\tau_{k}\right), k \in \mathcal{K}$, with $u(\cdot)$ denoting the Heaviside (unit) step function and $\tau_{k}$ the quantizer threshold. The quantized measurement $b_{k}$ is sent over a binary symmetric channel (BSC) and the DFC observes a (communication) errorprone $y_{k}$, that is $y_{k}=b_{k}$ with probability $1-P_{e, k}$ and $y_{k}=1-b_{k}$ with probability $P_{e, k}$, which we collect as $\boldsymbol{y} \triangleq\left[\begin{array}{lll}y_{1} & \cdots & y_{K}\end{array}\right]^{t}$. Here $P_{e, k}$ denotes the BEP of $k$ th link. The problem here is the derivation of a (computationally) simple test on the basis of $\boldsymbol{y}$ and the quantizer design for each sensor (i.e. an optimized $\tau_{k}, k \in \mathcal{K}$ ).

\section{RAO TEST}

\section{A. Test derivation}

A common approach to detection in composite hypothesis testing problems is given by the GLRT, which has been derived and studied in [10] for the model under investigation and whose expression is:

$$
\left\{\Lambda_{\mathrm{G}} \triangleq 2 \cdot \ln \left[\frac{P\left(\boldsymbol{y} ; \hat{\theta}_{1}\right)}{P\left(\boldsymbol{y} ; \theta_{0}\right)}\right]\right\} \underset{\substack{\hat{\mathcal{H}}=\mathcal{H}_{1} \\ \hat{\mathcal{H}}=\mathcal{H}_{0}}}{\gtrless} \gamma
$$

where $P(\boldsymbol{y} ; \theta)$ denotes the likelihood as a function of $\theta$, $\hat{\theta}_{1}$ is the maximum likelihood (ML) estimate under $\mathcal{H}_{1}$ (i.e. $\left.\hat{\theta}_{1} \triangleq \arg \max _{\theta} P(\boldsymbol{y} ; \theta)\right)$ and $\gamma$ is the threshold. It is clear from Eq. (2) that $\Lambda_{\mathrm{G}}$ requires the solution to an optimization problem; this increases the computational complexity of its implementation. However, in the special case $w_{k} \sim \mathcal{N}\left(0, \sigma_{k}^{2}\right)$ it was shown in [12] that ML estimation is a convex problem and thus it can be efficiently solved with local-optimization routines. Unfortunately a closed form for $\hat{\theta}_{1}$ is not available even under such an assumption.

As such, we pursue the derivation of the Rao test [11], which for the scalar case $(\theta \in \mathbb{R})$ is given implicitly in the form:

$$
\left\{\Lambda_{\mathrm{R}} \triangleq\left(\left.\frac{\partial \ln P(\boldsymbol{y} ; \theta)}{\partial \theta}\right|_{\theta=\theta_{0}}\right)^{2} / \mathrm{I}\left(\theta_{0}\right)\right\} \underset{\substack{\hat{\mathcal{H}}=\mathcal{H}_{1} \\ \gtrless}}{\gtrless} \gamma
$$

where $\mathrm{I}\left(\theta_{0}\right)$ is the Fisher information (FI), i.e. $\mathrm{I}(\theta) \triangleq$ $\mathbb{E}\left\{\left(\frac{\partial \ln [P(\boldsymbol{y} ; \theta)]}{\partial \theta}\right)^{2}\right\}$ evaluated at $\theta_{0}$. The motivation of our choice is the extreme simplicity of the test implementation (since $\hat{\theta}_{1}$ is not required, cf. Eq. (3)), but with the same weaksignal asymptotic performance as the GLRT, as supported from the theory [11].

\footnotetext{
${ }^{2}$ Noteworthy examples of such pdfs are the Gaussian, Laplace, Cauchy and generalized Gaussian distributions with zero mean [11].

${ }^{3}$ We restrict our attention to deterministic quantizers for simplicity; an alternative is the use of stochastic quantizers, however their analysis falls beyond the scope of this letter.
}

In order to obtain $\Lambda_{\mathrm{R}}$ explicitly, we expand $\ln [P(\boldsymbol{y} ; \theta)]$ as:

$$
\begin{gathered}
\ln [P(\boldsymbol{y} ; \theta)]=\sum_{k=1}^{K} \ln \left[P\left(y_{k} ; \theta\right)\right]= \\
\sum_{k=1}^{K}\left\{y_{k} \cdot \ln \left[\left(1-P_{e, k}\right) \alpha_{k}(\theta)+P_{e, k}\left(1-\alpha_{k}(\theta)\right)\right]+\right. \\
\left.\left(1-y_{k}\right) \cdot \ln \left[\left(1-P_{e, k}\right)\left(1-\alpha_{k}(\theta)\right)+P_{e, k} \alpha_{k}(\theta)\right]\right\}
\end{gathered}
$$

where $\alpha_{k}(\theta) \triangleq F_{w_{k}}\left(\tau_{k}-h_{k} \theta\right)$, with $F_{w_{k}}(\cdot)$ denoting the complementary cumulative distribution function of $w_{k}$. On the other hand, $\mathrm{I}(\theta)$ is given in closed form [10] as:

$$
\begin{aligned}
\mathrm{I}(\theta)= & \sum_{k=1}^{K}\left\{\frac{\left(1-2 P_{e, k}\right)^{2} \cdot h_{k}^{2} \cdot p_{w_{k}}^{2}\left(\tau_{k}-h_{k} \theta\right)}{P_{e, k}+\left(1-2 P_{e, k}\right) \cdot F_{w_{k}}\left(\tau_{k}-h_{k} \theta\right)} \times\right. \\
& \left.\frac{1}{\left[1-P_{e, k}-\left(1-2 P_{e, k}\right) \cdot F_{w_{k}}\left(\tau_{k}-h_{k} \theta\right)\right]}\right\} .
\end{aligned}
$$

Combining Eqs. (4) and (5) we obtain $\Lambda_{R}$ in closed form, as shown in Eq. (6) at the top of next page. It is apparent that $\Lambda_{\mathrm{R}}$ (as well as $\Lambda_{\mathrm{G}}$ ) is a function of $\tau_{k}, k \in \mathcal{K}$, which can be optimized in order to achieve (asymptotically) optimal performance.

\section{B. Quantizer design with asymptotic performance analysis}

We know from theory that $\Lambda_{\mathrm{R}}$ (as well as $\Lambda_{\mathrm{G}}$ ), is asymptotically (when the signal is weak 4 ) distributed as follows [11]:

$$
\Lambda_{\mathrm{R}} \stackrel{a}{\sim}\left\{\begin{array}{lll}
\chi_{1}^{2} & \text { under } & \mathcal{H}_{0} \\
\chi_{1}^{\prime 2}\left(\lambda_{Q}\right) & \text { under } & \mathcal{H}_{1}
\end{array}\right.
$$

where the non-centrality parameter $\lambda_{Q}$ is given by:

$$
\lambda_{Q} \triangleq\left(\theta_{1}-\theta_{0}\right)^{2} \mathrm{I}\left(\theta_{0}\right)
$$

with $\theta_{1}$ being the true value under $\mathcal{H}_{1}$. Clearly the larger $\lambda_{Q}$, the better the GLRT and Rao tests will perform. Also, as shown in [10], $\lambda_{Q}$ is a function of $\tau_{k}, k \in \mathcal{K}$; therefore we choose $\tau_{k}, k \in \mathcal{K}$, in order to maximize $\lambda_{Q}$, that is

$$
\begin{aligned}
\arg \max _{\left\{\tau_{1}, \ldots, \tau_{K}\right\}} & \left\{\lambda_{Q}=\theta^{2} \sum_{k=1}^{K}\left[\frac{\left(1-2 P_{e, k}\right)^{2} \cdot h_{k}^{2} \cdot p_{w_{k}}^{2}\left(\tau_{k}\right)}{P_{e, k}+\left(1-2 P_{e, k}\right) \cdot F_{w_{k}}\left(\tau_{k}\right)} \times\right.\right. \\
& \left.\left.\frac{1}{1-P_{e, k}-\left(1-2 P_{e, k}\right) \cdot F_{w_{k}}\left(\tau_{k}\right)}\right]\right\}
\end{aligned}
$$

which can be decoupled into the following set of $K$ independent threshold design problems:

$$
\arg \max _{\tau_{k}}\left\{g_{k}\left(\tau_{k}\right) \triangleq \frac{p_{w_{k}}^{2}\left(\tau_{k}\right)}{\Delta_{k}+F_{w_{k}}\left(\tau_{k}\right) \cdot\left[1-F_{w_{k}}\left(\tau_{k}\right)\right]}\right\}
$$

where $\Delta_{k} \triangleq\left[P_{e, k} \cdot\left(1-P_{e, k}\right)\right] /\left(1-2 P_{e, k}\right)^{2}$. It is known from quantized estimation literature [13], [14] that many unimodal and symmetric $p_{w_{k}}(\cdot)$ 's with $\mathbb{E}\left\{w_{k}\right\}=0$ lead to $\tau_{k}^{*} \triangleq \arg \max _{\tau_{k}} g_{k}\left(\tau_{k}\right)=0$ (independent of $\Delta_{k}$ ); such examples are the Gaussian, Laplace, Cauchy and the widely used generalized normal distribution, that is $p_{w_{k}}\left(\tau_{k}\right)=$

\footnotetext{
${ }^{4}$ That is $\left|\theta_{1}-\theta_{0}\right|=c / \sqrt{K}$ for some constant $c>0$ [11].
} 


$$
\Lambda_{\mathrm{R}}=\left(\sum_{k=1}^{K} \frac{\left(1-2 \cdot P_{e, k}\right) \cdot h_{k} \cdot p_{w_{k}}\left(\tau_{k}\right) \cdot\left[2 y_{k}-1\right]}{\left(1-P_{e, k}\right) \cdot F_{w_{k}}\left(\tau_{k}\right)^{y_{k}} \cdot\left[1-F_{w_{k}}\left(\tau_{k}\right)\right]^{1-y_{k}}+P_{e, k} \cdot F_{w_{k}}\left(\tau_{k}\right)^{1-y_{k}} \cdot\left[1-F_{w_{k}}\left(\tau_{k}\right)\right]^{y_{k}}}\right)^{2} \times(\mathrm{I}(\theta=0))^{-1}
$$

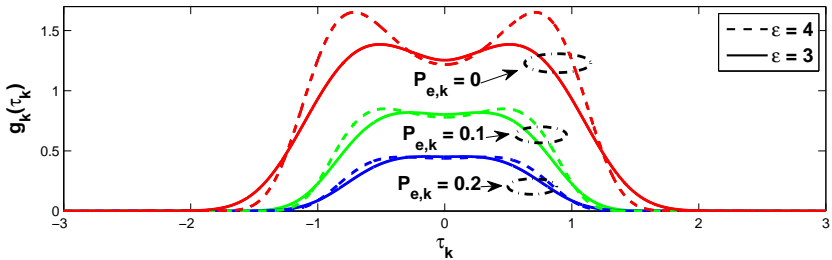

Figure 1. Effect of $P_{e, k}$ on $g_{k}\left(\tau_{k}\right)$ when $p_{w_{k}}\left(\tau_{k}\right)=$ $\frac{\epsilon}{2 \alpha \Gamma(1 / \epsilon)} \exp \left[-\left(\frac{\left|\tau_{k}\right|}{\alpha}\right)^{\epsilon}\right] ; \alpha=1, \epsilon \in\{3,4\}$ and $P_{e, k} \in\{0,0.1,0.2\}$.

$\frac{\epsilon}{2 \alpha \Gamma(1 / \epsilon)} \exp \left[-\left(\frac{\left|\tau_{k}\right|}{\alpha}\right)^{\epsilon}\right]$, only when $0 \leq \epsilon \leq 2$; on the other hand when $\epsilon>2, g_{k}\left(\tau_{k}\right)$ becomes bimodal (since it is symmetric) as shown in Fig. 1 However the effect of a nonideal BSC smoothes the gain achieved by $\tau_{k}^{*}$ and thus $\tau_{k}=0$ is still a good (sub-optimal) choice. Substituting $\tau_{k}=0, k \in \mathcal{K}$, in Eq. (6), leads to the following simplified expression for threshold-optimized Rao test (denoted with $\Lambda_{\mathrm{R}}^{*}$ ):

$$
\Lambda_{\mathrm{R}}^{*}=\frac{4 \cdot\left[\sum_{k=1}^{K}\left(1-2 P_{e, k}\right) \cdot p_{w_{k}}(0) \cdot h_{k} \cdot\left(y_{k}-\frac{1}{2}\right)\right]^{2}}{\sum_{k=1}^{K}\left(1-2 P_{e, k}\right)^{2} \cdot p_{w_{k}}^{2}(0) \cdot h_{k}^{2}}
$$

which is considerably simpler than the GLRT, as it obviates solution of an optimization problem (which depends on $\left.p_{w_{k}}(\cdot)\right)$. Furthermore, the corresponding optimized noncentrality parameter, denoted with $\lambda_{Q}^{*}$, is given by:

$$
\lambda_{Q}^{*}=4 \theta^{2} \cdot \sum_{k=1}^{K}\left[\left(1-2 P_{e, k}\right)^{2} \cdot p_{w_{k}}^{2}(0) \cdot h_{k}^{2}\right]
$$

Remarks - In the case of BSCs of the same quality (i.e. $P_{e, k}=$ $\left.P_{e}, k \in \mathcal{K}\right)$ we simply get $\lambda_{Q}^{*}=\left(1-2 P_{e}\right)^{2} \cdot \lambda_{Q_{0}}^{*}$, where $\lambda_{Q_{0}}^{*} \triangleq 4 \theta^{2} \cdot \sum_{k=1}^{K}\left[p_{w_{k}}^{2}(0) \cdot h_{k}^{2}\right]$ represents $\lambda_{Q}^{*}$ in the ideal $\mathrm{BSC}$ case $\left(P_{e, k}=0, k \in \mathcal{K}\right)$. This result generalizes the one in [10], by stating that the loss due to non-ideal communications is asymptotically independent of $p_{w_{k}}(\cdot), k \in \mathcal{K}$.

\section{COMPARISON IN HOMOGENEOUS SCENARIO}

In this section we study the simplified scenario $h_{k}=h$, $p_{w_{k}}(\cdot)=p_{w}(\cdot), P_{e, k}=P_{e}, k \in \mathcal{K}$, to get an intuitive interpretation of the two threshold-optimized tests $\left(\tau_{k}^{*}=0\right)$. Based on these assumptions, the statistics in Eqs. (2) and (11) reduce to:

$$
\begin{aligned}
\Lambda_{\mathrm{G}}^{*} & =2 K \cdot\left[\hat{\rho} \ln \left(\frac{\hat{\rho}}{\rho_{0}}\right)+(1-\hat{\rho}) \ln \left(\frac{1-\hat{\rho}}{1-\rho_{0}}\right)\right] \\
& =2 K \cdot D_{\mathrm{KL}}\left(\hat{P}\left(y_{k}\right) \| P\left(y_{k} ; \theta_{0}\right)\right) \\
\Lambda_{\mathrm{R}}^{*} & =4 K \cdot\left[\hat{\rho}-\rho_{0}\right]^{2} \\
& =4 K \cdot\left[D_{\mathrm{TVD}}\left(\hat{P}\left(y_{k}\right) \| P\left(y_{k} ; \theta_{0}\right)\right)\right]^{2}
\end{aligned}
$$

where $\Lambda_{\mathrm{G}}^{*} \triangleq \Lambda_{\mathrm{G}}\left(\tau_{k}=0\right), \hat{\rho} \triangleq \sum_{k=1}^{K} y_{k} / K$ and $\rho_{0} \triangleq 1 / 2$. Here $\hat{P}\left(y_{k}\right)$ represents the empirical distribution of the i.i.d. binary source $\left\{y_{1}, \ldots, y_{K}\right\}$ and $D_{\mathrm{KL}}(\cdot \| \cdot)$ and $D_{\mathrm{TVD}}(\cdot \| \cdot)$ denote the Kullback-Leibler (KL) and total variation distance (TVD) divergences, respectively [15]. It is worth noticing that in Eq. (14) we exploited the closed form of $\hat{\theta}_{1}=$ $-\frac{1}{h} F_{w}^{-1}\left(\left(\hat{\rho}-P_{e}\right) /\left(1-2 P_{e}\right)\right)$ (see [12] for a similar result).

Exploiting KL 5 and TVD divergences properties it can be shown that both Eqs. (14) and (16) are monotone (increasing) functions of $\left|\hat{\rho}-\rho_{0}\right|$ and therefore represent equivalent tests in a homogeneous sensor scenario, meaning their performances coincide also for a finite number of sensors.

Finally, we derive a tighter asymptotic form of the conditional pdf (not requiring the weak-signal assumption) of both the tests in this scenario with the help of the central limit theorem (CLT) [15]. Without loss of generality we focus hereinafter on $\Lambda_{\mathrm{R}}^{*}$ (since $\Lambda_{\mathrm{G}}^{*}$ has the same performance). For this purpose, we define the RV $\xi \triangleq \frac{\sum_{k=1}^{K}\left(2 y_{k}-1\right)}{\sqrt{K}}$ and we consider the asymptotic form of $p_{\xi}\left(\cdot \mid \mathcal{H}_{i}\right), i \in\{0,1\}$, which according to the CLT is given as $K \rightarrow+\infty$ by:

$$
\xi\left|\mathcal{H}_{0} \stackrel{a}{\sim} \mathcal{N}(0,1) \quad \xi\right| \mathcal{H}_{1} \stackrel{a}{\sim} \mathcal{N}\left(\sqrt{K} \tilde{\mu}_{1}, \tilde{\sigma}_{1}^{2}\right)
$$

where $\tilde{\mu}_{1} \triangleq\left(1-2 P_{e}\right)\left(2 \rho_{1}-1\right), \tilde{\sigma}_{1}^{2} \triangleq 4 \cdot\left[1+P_{e}\left(2 \rho_{1}-1\right)-\right.$ $\left.\rho_{1}\right] \cdot\left[\rho_{1}+\left(1-2 \rho_{1}\right) P_{e}\right]$ and $\rho_{1} \triangleq F_{w}(-h \theta)$. From inspection of Eq. (15), it can be readily verified that $\Lambda_{\mathrm{R}}^{*}=\xi^{2}$ holds, which can be exploited to obtain closed form performance expressions.

\section{Numerical Results}

In this section we compare the Rao test to the GLRT. We evaluate the performance in terms of system false alarm and detection probabilities, defined as $P_{F_{0}} \triangleq \operatorname{Pr}\left\{\Lambda>\gamma \mid \mathcal{H}_{0}\right\}$ and $P_{D_{0}} \triangleq \operatorname{Pr}\left\{\Lambda>\gamma \mid \mathcal{H}_{1}\right\}$, respectively, where $\Lambda$ is the statistic employed at the DFC. We also define the $k$ th sensor observation signal-to-noise ratio (SNR) as $\Gamma_{k} \triangleq\left(h_{k}^{2} \theta^{2} / \mathbb{E}\left\{w_{k}^{2}\right\}\right)$.

In Fig. 2 we illustrate $P_{D_{0}}$ vs $P_{F_{0}}$ in a WSN with $K=5$ sensors where $\theta=1, h_{k} \sim U(0, a), k \in \mathcal{K}$ (but known at the DFC), and two noise pdfs: $(i) w_{k} \sim \mathcal{N}\left(0, \sigma_{k}^{2}\right)$ and (ii) $w_{k} \sim \mathcal{L}\left(0, \beta_{k}\right)$, such that $\mathbb{E}\left\{w_{k}^{2}\right\}=1$. We consider four combinations corresponding to $P_{e, k}=P_{e} \in\{0,0.2\}$ and $\bar{\Gamma}_{d B} \in\{0,10\}$, where we have denoted $\bar{\Gamma} \triangleq \mathbb{E}\left\{\Gamma_{k}\right\}$ (in our case $\left.\bar{\Gamma}=\left(a^{2} \theta^{2}\right) / 3 \cdot \mathbb{E}\left\{w_{k}^{2}\right\}\right)$ as the average observation SNR. The figures are based on $10^{5}$ Monte Carlo runs. First, it is apparent that the performances of the GLR and the Rao tests are practically the same for all the considered scenarios; however the implementation of the Rao test is much simpler than that of the GLRT. Also, the difference in performances under Laplacian and Gaussian noises is significant only at $\bar{\Gamma}_{d B}=0$, while at $\bar{\Gamma}_{d B}=10$ the curves almost overlap. This is explained since when $\bar{\Gamma}$ is low the signal is more concentrated around zero. Then the imbalance in the binary pmf observed at the output of each quantizer is higher when $w_{k} \sim \mathcal{L}\left(0, \beta_{k}\right)$.

\footnotetext{
${ }^{5}$ Since it is increasing when $\hat{\rho}>\rho_{0}$ and symmetric around $\rho_{0}$.
} 

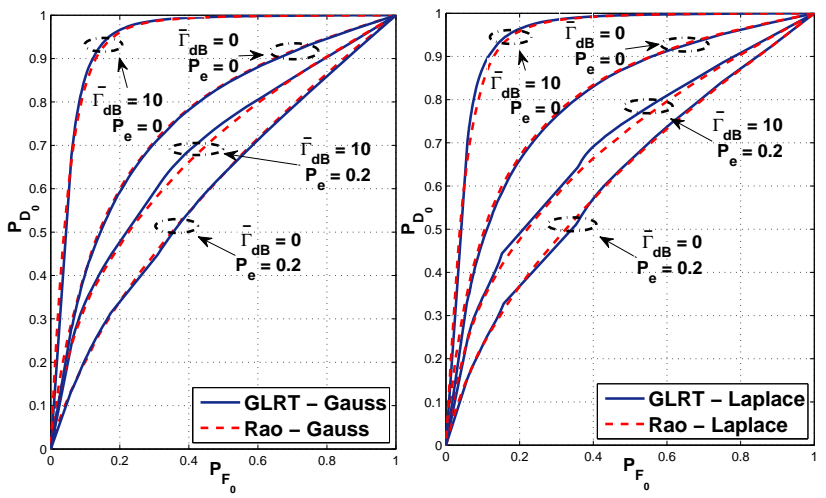

Figure 2. $P_{D_{0}}$ vs $P_{F_{0}}$; WSN with $K=5$ sensors, $h_{k} \sim \mathcal{U}(0, a), \theta=1$, $\mathbb{E}\left\{w_{k}^{2}\right\}=1$ for Gaussian and Laplace noise; $P_{e} \in\{0,0.2\}, \bar{\Gamma}_{d B} \in\{0,10\}$.

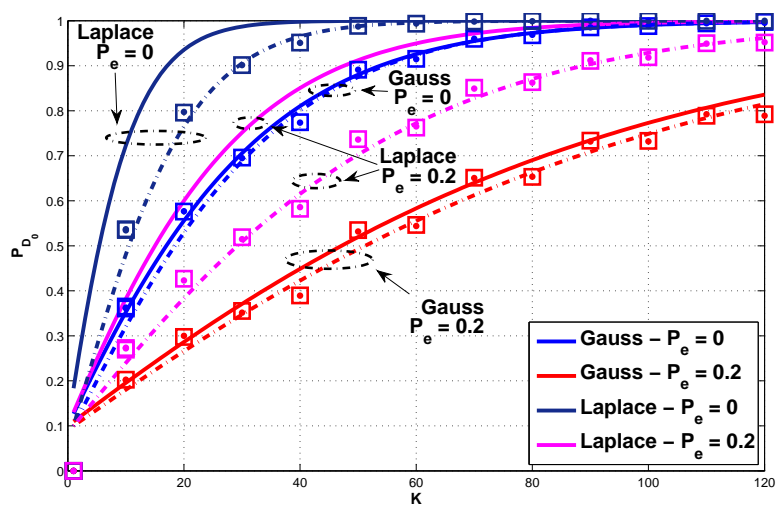

Figure 3. $\quad P_{D_{0}}$ vs $K ; P_{F_{0}}=0.1$. Setup: $\theta=0.5 ; h_{k}=1, \mathbb{E}\left\{w_{k}^{2}\right\}=1$ $\left(\left(\Gamma_{k}\right)_{d B} \approx-6\right), P_{e, k} \in\{0,0.2\}, k \in \mathcal{K}$ (homogeneous scenario). Square $(\square)$ and bullet $(\bullet)$ markers refer to GLRT and Rao test, respectively; solid and dash-dot lines refer to weak-signal and CLT-based asymptotic pdfs, respectively.

In Fig. 3 we show $P_{D_{0}}$ as a function of $K$, assuming $P_{F_{0}}=0.1$. We consider $\theta=0.5, h_{k}=1$ and two noise pdfs: $($ i $) w_{k} \sim \mathcal{N}\left(0, \sigma_{k}^{2}\right)$ and $(i i) w_{k} \sim \mathcal{L}\left(0, \beta_{k}\right)$, such that $\mathbb{E}\left\{w_{k}^{2}\right\}=1$ (thus $\left(\Gamma_{k}\right)_{d B} \approx-6$ ), $k \in \mathcal{K}$. Also, we consider $P_{e} \in\{0,0.2\}$, thus determining a homogeneous scenario. First, Monte Carlo simulations confirm the theoretical coincidence between the Rao test (bullet markers) and the GLRT (square markers). Secondly, it is apparent that the CLT-based performance expressions (dash-dot) are as accurate as those based on the weak-signal assumption (solid lines) for Gaussian noise, while in the Laplacian Case the weak-signal distribution is far from being representative of the distribution. Interestingly, when $P_{e}=0, \lambda_{Q_{0}}^{*}$ in the Laplacian case coincides with the non-centrality parameter achieved by a GLRT (or Rao test) based on the raw $x_{k}, k \in \mathcal{K}$, given by $\lambda_{U Q}=\theta^{2} \sum_{k=1}^{K} \frac{h_{k}^{2}}{\beta_{k}^{2}}$, that is Eq. (12) does not predict the loss due to quantization. On the other hand, by exploiting the CLT-based performance in Eq. (17), we can compare $\lambda_{U Q}$ with the modified deflection coefficient of the asymptotic problem given by Eq. (17) $d_{Q} \triangleq K \tilde{\mu}_{1}^{2} / \tilde{\sigma}_{1}^{2}$, which for the Laplacian noise is given by $d_{Q}=\frac{K \theta^{2}\left(h^{2} / \beta^{2}\right)}{[1-\exp (-|h \theta| / \beta)]^{2}} \cdot\left[1-\left(1-\exp \left(-\frac{|h \theta|}{\beta}\right)^{2}\right]\right.$; thus for this problem we have $\lambda_{U Q} / d_{Q} \approx 1.45$, which predicts the performance loss well.

\section{CONClusions}

We studied the Rao test for decentralized detection with an unknown deterministic signal as an attractive alternative to GLRT for a general model with quantized measurements, zeromean, unimodal and symmetric noise (pdf), non-ideal and nonidentical BSCs. The asymptotically optimal sensor thresholds were shown to be zero for many pdfs of interest and a fair choice in other scenarios; this result was exploited to simplify further the Rao test formula. Furthermore, it was shown through simulations that the Rao test, in addition to being asymptotically equivalent to the GLRT, achieves practically the same performance in the finite number of sensors case; for the case of homogeneous sensors a theoretical coincidence of the two tests was established. In such a scenario a general asymptotic performance were derived based on the CLT and not requiring the weak-signal assumption. These latter were shown to be crucial in performance analysis with peaked noise pdfs.

\section{REFERENCES}

[1] J. N. Tsitsiklis, "Decentralized detection," Advances in Statistical Signal Processing, vol. 2, no. 2, pp. 297-344, 1993.

[2] R. Viswanathan and P. K. Varshney, "Distributed detection with multiple sensors - Part I: Fundamentals," Proc. IEEE, vol. 85, no. 1, pp. 54-63, Jan. 1997.

[3] V. A. Aalo and R. Viswanathan, "Multilevel quantisation and fusion scheme for the decentralised detection of an unknown signal," Proc. of IEE Radar, Sonar and Navig., vol. 141, no. 1, pp. 37-44, Feb. 1994.

[4] B. Chen, R. Jiang, T. Kasetkasem, and P. K. Varshney, "Channel aware decision fusion in wireless sensor networks," IEEE Trans. Signal Process., vol. 52, no. 12, pp. 3454-3458, Dec. 2004.

[5] R. Niu and P. K. Varshney, "Performance analysis of distributed detection in a random sensor field," IEEE Trans. Signal Process., vol. 56, no. 1, pp. 339-349, Jan. 2008.

[6] D. Ciuonzo, G. Romano, and P. Salvo Rossi, "Channel-aware decision fusion in distributed MIMO wireless sensor networks: Decode-and-fuse vs. decode-then-fuse," IEEE Trans. Wireless Commun., vol. 11, no. 8, pp. 2976-2985, Aug. 2012.

[7] D. Ciuonzo, G. Romano, and P. Salvo Rossi, "Optimality of received energy in decision fusion over Rayleigh fading diversity MAC with nonidentical sensors," IEEE Trans. Signal Process., vol. 61, no. 1, pp. 22-27, Jan. 2013.

[8] R. Niu and P. K. Varshney, "Joint detection and localization in sensor networks based on local decisions," in Fortieth Asilomar Conference on Signals, Systems and Computers, 2006, pp. 525-529.

[9] S. G. Iyengar, R. Niu, and P. K. Varshney, "Fusing dependent decisions for hypothesis testing with heterogeneous sensors," IEEE Trans. Signal Process., vol. 60, no. 9, pp. 4888-4897, Sep. 2012.

[10] J. Fang, Y. Liu, H. Li, and S. Li, "One-bit quantizer design for multisensor GLRT fusion," IEEE Signal Process. Lett., vol. 20, no. 3 , pp. 257-260, Mar. 2013.

[11] S. M. Kay, Fundamentals of Statistical Signal Processing, Volume 2: Detection Theory. Prentice Hall PTR, Jan. 1998.

[12] A. Ribeiro and G. B. Giannakis, "Bandwidth-constrained distributed estimation for wireless sensor networks - Part I: Gaussian case," IEEE Trans. Signal Process., vol. 54, no. 3, pp. 1131-1143, Mar. 2006.

[13] H. C. Papadopoulos, G. W. Wornell, and A. V. Oppenheim, "Sequential signal encoding from noisy measurements using quantizers with dynamic bias control," IEEE Trans. Inf. Theory, vol. 47, no. 3, pp. 9781002, Mar. 2001

[14] D. Rousseau, G. V. Anand, and F. Chapeau-Blondeau, "Nonlinear estimation from quantized signals: Quantizer optimization and stochastic resonance," in Proc. 3rd Int. Symp. Physics in Signal and Image Processing, 2003, pp. 89-92.

[15] T. M. Cover and J. A. Thomas, Elements of Information Theory. WileyInterscience, 2006. 